\title{
A REMARK ON THE NUMBER OF DISTINCT PRIME DIVISORS OF INTEGERS
}

\author{
MEHDI HASSANI
}

\begin{abstract}
We study the asymptotic formula for the sum $\sum_{n \leqslant x} \omega(n)$ where $\omega(n)$ denotes the number of distinct prime divisors of $n$, and we perform some computations which detect curve patterns in the distribution of a related sequence.
\end{abstract}

\section{Introduction}

Let $\omega(n)=\sum_{p \mid n} 1$ be the number of distinct prime divisors of the positive integer $n$. In 1917, Hardy and Ramanujan [2] proved the following average result

$$
\sum_{n \leqslant x} \omega(n)=x \log \log x+M x+O\left(\frac{x}{\log x}\right),
$$

where $M$ is known as the Meissel-Mertens constant [1] and defined by

$$
M=\gamma+\sum_{p}\left(\log \left(1-p^{-1}\right)+p^{-1}\right) \approx 0.261497212847642783755426838609,
$$

and $\gamma$ refers to Euler's constant. In the present note we improve the error term in (1), by evaluating the exact value of $O$-term. Indeed we prove the following.

Theorem 1. As $x \rightarrow \infty$ one has

$$
\sum_{n \leqslant x} \omega(n)=x \log \log x+M x-(1-\gamma) \frac{x}{\log x}+O\left(\frac{x}{\log ^{2} x}\right) .
$$

Remark 2. To clear more details of the asymptotic relation (2) we consider the function

$$
\mathscr{R}(x)=\frac{\log x}{x}\left(\sum_{n \leqslant x} \omega(n)-x \log \log x-M x\right) .
$$

While the truth of Theorem 1 implies that $\mathscr{R}(x) \rightarrow \gamma-1$ as $x \rightarrow \infty$, we perform computations which detect curve patterns in the distribution of the points $(n, \mathscr{R}(n))$. For integers $n$ with $2 \leqslant n \leqslant 82$ we have $\mathscr{R}(n)>1-\gamma$. There are some partial curve patters, as pictured in Figures 1 and 2, waiting for a mathematical justification. It seems that for $n \geqslant 343$ one has $\mathscr{R}(n)<1-\gamma$, and the minimum value of $\mathscr{R}(n)$ occurs at $n=1879$.

Received September 25, 2015, accepted November 16, 2016. 2010 Mathematics Subject Classification. 11A41, 11A25.

Key words and phrases. Prime number, arithmetic function. 

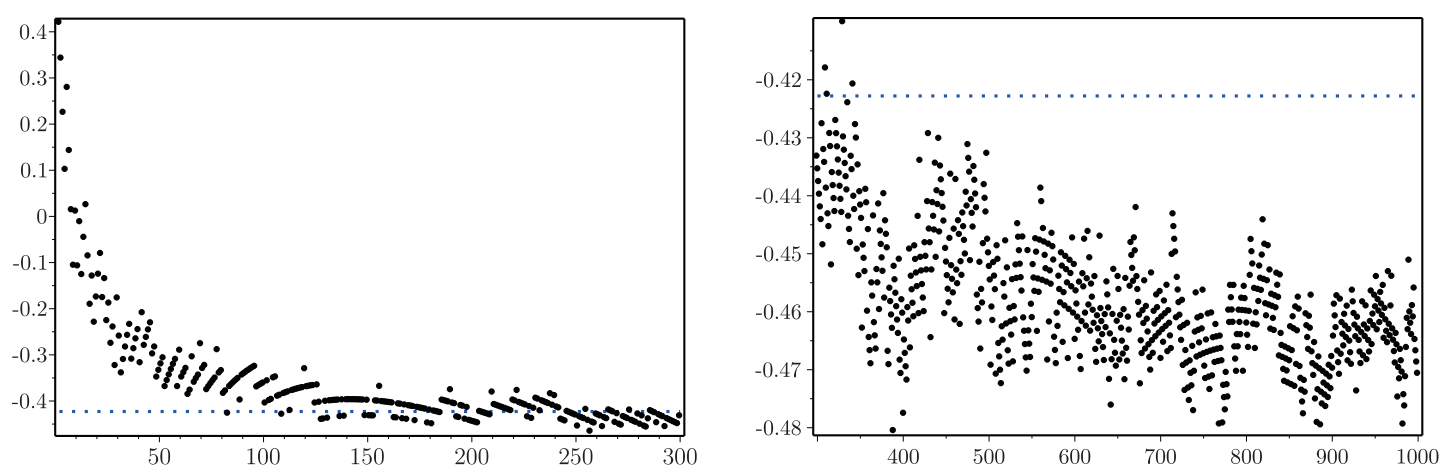

Figure 1: Graphs of the points $(n, \mathscr{R}(n))$ for $2 \leqslant n \leqslant 1000$, and the dotted line $y=\gamma-1$.

Proof of Theorem 1. Let us denote integer part and fractional part of the real number $x$ by $[x]$ and $\{x\}$, respectively. Also, let

$$
\mathscr{A}(x)=\sum_{p \leqslant x} \frac{1}{p}, \quad \text { and } \quad \mathscr{F}(x)=\sum_{p \leqslant x}\left\{\frac{x}{p}\right\} .
$$

We have

$$
\sum_{n \leqslant x} \omega(n)=\sum_{n \leqslant x} \sum_{p \mid n} 1=\sum_{p \leqslant x} \sum_{\substack{n \leqslant x \\ p \mid n}} 1=\sum_{p \leqslant x}\left[\frac{x}{p}\right]=\sum_{p \leqslant x}\left(\frac{x}{p}-\left\{\frac{x}{p}\right\}\right),
$$

and hence

$$
\sum_{n \leqslant x} \omega(n)=x \mathscr{A}(x)-\mathscr{F}(x) .
$$

The sum $\mathscr{F}(x)$ on the fractional parts has been studied by de la Vallée Poussin [5], where he showed by elementary methods that $\mathscr{F}(x) \sim(1-\gamma) \frac{x}{\log x}$ as $x \rightarrow \infty$. More precisely, by using Perron's formula, Lee [3] proved that

$$
\sum_{p^{\alpha} \leqslant x}\left\{\frac{x}{p^{\alpha}}\right\}=(1-\gamma) \frac{x}{\log x}+O\left(\frac{x}{\log ^{2} x}\right) .
$$

The difference of the later sum by $\mathscr{F}(x)$ is not large, because

$$
\sum_{p^{\alpha} \leqslant x}\left\{\frac{x}{p^{\alpha}}\right\}-\mathscr{F}(x)=\sum_{\substack{p^{\alpha} \leqslant x \\ \alpha \geqslant 2}}\left\{\frac{x}{p^{\alpha}}\right\} \ll \sum_{\substack{p^{\alpha} \leqslant x \\ \alpha \geqslant 2}} 1 \ll \sqrt{x} \log ^{2} x .
$$

Thus, we get

$$
\mathscr{F}(x)=(1-\gamma) \frac{x}{\log x}+O\left(\frac{x}{\log ^{2} x}\right) .
$$

We recall that Theorem 5 of [4] implies

$$
\mathscr{A}(x):=\sum_{p \leqslant x} \frac{1}{p}=\log \log x+M+O\left(\frac{1}{\log ^{2} x}\right) .
$$

Finally, we combine (4) with (5) and (6) to deduce (2). 

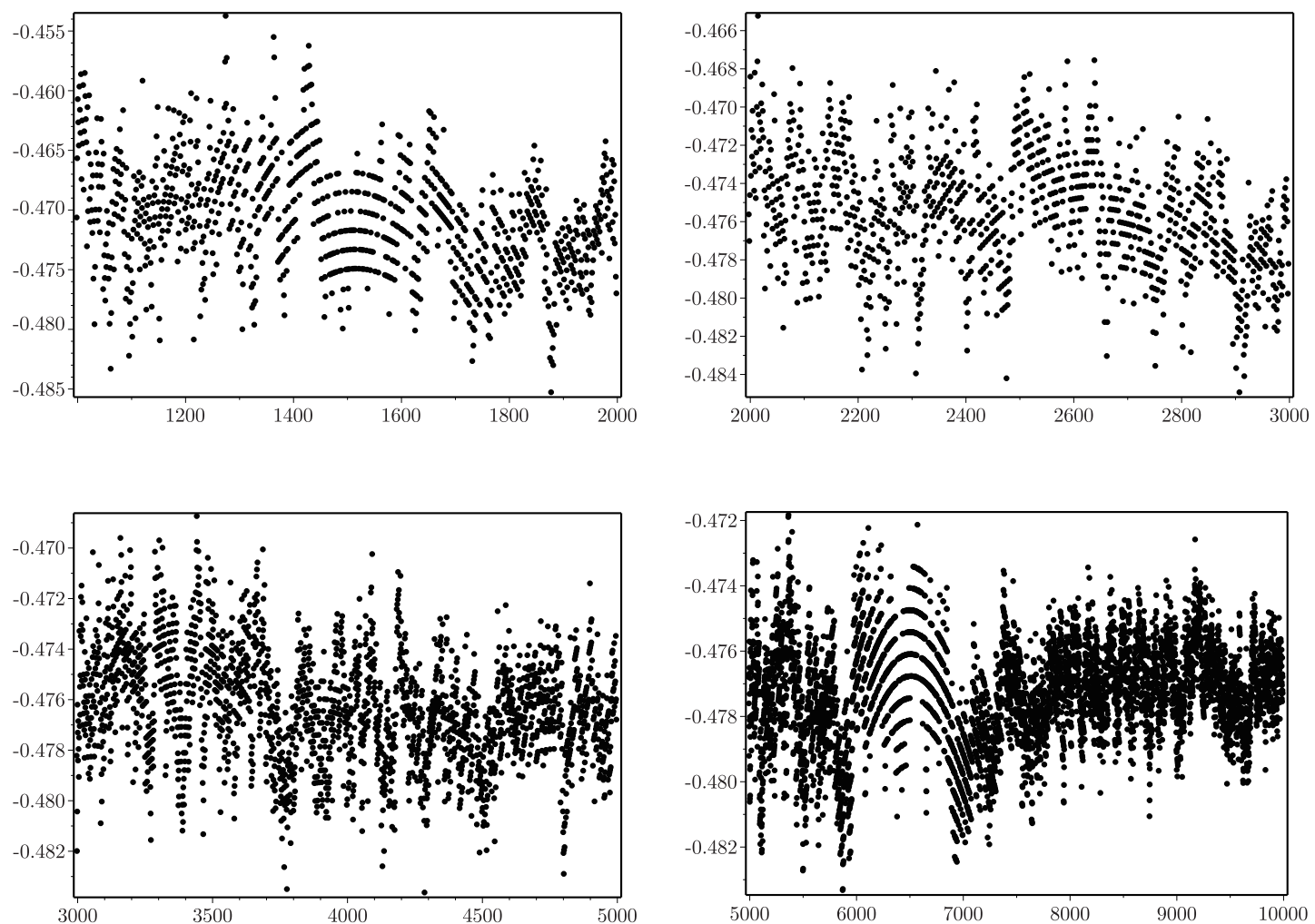

Figure 2: Graphs of the points $(n, \mathscr{R}(n))$ for $10^{3} \leqslant n \leqslant 10^{4}$.

\section{References}

[1] S. R. Finch, Mathematical constants, Encyclopedia of Mathematics and its Applications, 94, Cambridge University Press, Cambridge, 2003.

[2] G. H. Hardy and S. Ramanujan, The normal number of prime factors of a number n, Quart. J. Math., 48 (1917), 76-92.

[3] J. Lee, The second central moment of additive functions, Proc. Amer. Math. Soc., 114 (1992), 887-895.

[4] J. B. Rosser and L. Schoenfeld, Approximate formulas for some functions of prime numbers, Illinois J. Math., 6 (1962), 64-94.

[5] C. de la Vallée Poussin, Sur les valeurs moyennes de certaines fonctions arithmétiques, Annales de la société scientifique de Bruxelles, 22 (1898), 84-90.

Department of Mathematics, University of Zanjan, University Blvd., 45371-38791, Zanjan, Iran.

E-mail: mehdi.hassani@znu.ac.ir 